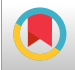

\title{
Prevalence of Coronary Artery Ectasia and Associated Risk Factors
}

\author{
Seyyed Ali Moezi Bady (iD) ${ }^{1}$, Elham Rezvanian ${ }^{2}$, Nahid Azdaki (iD ${ }^{1}$, Saeede Khosravi Bizhaem (iD) ${ }^{3}$ and \\ Toba Kazemi (iD) 1, 4, ${ }^{*}$ \\ ${ }^{1}$ Cardiovascular Diseases Research Center, Department of Cardiology, School of Medicine, Birjand University of Medical Sciences, Birjand, Iran \\ ${ }^{2}$ Student Research Committee, Birjand University of Medical Sciences, Birjand, Iran \\ ${ }^{3}$ Cardiovascular Diseases Research Center, Birjand University of Medical Sciences, Birjand, Iran \\ ${ }^{4}$ Razi Clinical Research Development Unit (RCRDU), Birjand University of Medical Sciences (BUMS), Birjand, Iran \\ "Corresponding author: Cardiovascular Diseases Research Center, Department of Cardiology, School of Medicine, Birjand University of Medical Sciences, Birjand, Iran. Email: \\ drtooba.kazemi@gmail.com
}

Received 2020 April 22; Revised 2020 June 02; Accepted 2020 June 03.

\begin{abstract}
Objectives: This study aimed to evaluate the incidence of coronary artery ectasia (CAE) and its associated factors in the East of Iran. Methods: A cross-sectional study was conducted on 2,795 patients undergoing coronary angiography between 2011 and 2017. Patients were categorized into three groups: Coronary artery ectasia, normal coronary artery, and coronary artery stenosis. Patients' demographic data, cardiac risk factors, and angiographic results were extracted from medical records. Then, information on CAE patients was evaluated. Data were analyzed by the independent t-test, Mann-Whitney test, and chi-square test. Statistical significance was defined by a P-value $\leq 0.05$.

Results: The prevalence of CAE was 3.04\%. The mean age of CAE patients ( $59.44 \pm 10.15)$ was significantly higher than that of normal coronary artery patients ( $53.98 \pm 9.97)$. The proportion of men was significantly lower in the CAE group ( $48.2 \%)$ than in the CAS group (62.1\%) but higher than in the normal group (32.3). Body mass index (BMI) and low HDL (high-density lipoprotein) were higher in the CAE group, but diabetes mellitus was lower than in the CAS group.

Conclusions: The prevalence of CAE was low in our study. Old age, male sex, obesity, and low HDL were CAE risk factors, but diabetes mellitus was a preventing factor in our study.
\end{abstract}

Keywords: Cardiovascular Disease, Coronary Artery Ectasia, Diabetes Mellitus, Prevalence

\section{Background}

Cardiovascular disease, especially coronary artery disease (CAD), is a major public health problem and the main cause of death in developing countries. The most common manifestation of coronary artery involvement is stenosis, but in some cases, abnormal dilatation of the coronary artery may also occur (1). Coronary artery ectasia (CAE) can be defined as diffuse or local dilatation of the coronary artery as 1.5 times more than the wider portion of the adjacent vascular lumen (2).

Atherosclerosis is considered the main etiologic factor responsible for $50 \%$ of the CAE cases in young people. Kawasaki's disease is the most common cause of CAE in children and young adults. It seems that CAE is a result of excessive remodeling due to dropped enzymatic activity of extracellular matrix and thickening of the media layer of blood vessels. Other etiologies for CAE include congenital malformations, chest trauma, connective tissue disorder, collagenopathies such as Marfan syndrome, and primary hyperaldosteronism $(3,4)$.

The prevalence of CAE has been reported in several studies as follows: $4.3 \%$ in Turkey, $3.9 \%$ in Spain, $7.2 \%$ in Greece, and 1.4\% in the United Kingdom (1). It is believed that CAE clinical symptoms depend on the presence of obstructive cardiovascular disease. However, in the absence of significant coronary stenosis, CAE may cause angina and myocardial ischemia during exercise and even myocardial infarction (5).

\section{Objectives}

This study aimed to determine the prevalence and risk factors of CAE with atherosclerosis.

\section{Methods}

Data were gathered from 2,795 patients who underwent coronary angiography by two cardiologists from April 2011 to March 2017 in Vali-e-Asr and Razi Hospitals in 
Birjand, East of Iran. Demographic and cardiac risk factors were obtained from medical records. Cardiac risk factors were defined as follows: Cigarette smoking: Patients who regularly smoked cigarettes one or more times per day or had quit smoking during the last 24 months; Dyslipidemia: Total cholesterol $\geq 240 \mathrm{mg} / \mathrm{dL}$ or $\mathrm{LDL} \geq 130$ $\mathrm{mg} / \mathrm{dL}$ or triglycerides $\geq 150 \mathrm{mg} / \mathrm{dL}$ or $\mathrm{HDL}<40 \mathrm{mg} / \mathrm{dL}$ in males and $<50 \mathrm{mg} / \mathrm{dL}$ in females; Family history of CAD: CAD in the first-degree relatives before the age of 55 years in men and 65 years in women; hypertension: History of HTN or BP $\geq 140 / 90 \mathrm{mmHg}$; and DM: History of diabetes or fasting blood sugar (FBS) $\geq 126 \mathrm{mg} / \mathrm{dL}$. The body mass index (BMI) was calculated via the formula of weight $(\mathrm{kg}) /$ squared height $\left(\mathrm{m}^{2}\right)$, and all patients with a BMI $\geq 25$ $\mathrm{kg} / \mathrm{m}^{2}$ were identified as overweight or obese.

According to the results of angiography, the patients were categorized into three groups: 1) Normal coronary artery: Coronary artery without any stenosis, 2) CAE: Coronary artery luminal diameter $>1.5$ times the adjacent normal segment without any coronary artery stenosis, and 3) coronary artery stenosis (CAS): Coronary artery with at least 50\% luminal narrowing without CAE.

\subsection{Statistical Analysis}

Data were analyzed by SPSS-22.0 for Windows (SPSS Inc., Chicago, IL). Continuous variables are presented as mean \pm standard deviation (SD), and the categorical variables are summarized by percentages. The continuous variables were compared using the Mann-Whitney U, and the categorical variables were compared using the chi-squared test. Statistical significance was defined by a P-value $\leq$ 0.05 .

\section{Results}

We categorized the patients into three groups: 1966 patients in the CAS group, 744 in the normal group, and $85(3.04 \%)$ patients in the CAE group (male/female ratio of 44:41). Table 1 compares the three groups for demographic and clinical characteristics.

The prevalence of CAE among male patients was 2.7\% (41/1501). The proportion of men was significantly lower in the CAE group than in the CAS group but was higher than in the normal group. As shown in the table, the mean age of CAE patients ( $59.44 \pm 10.15)$ was similar to the age in the CAS group ( $61.19 \pm 10.42)$ but significantly higher than the age of people with normal coronary arteries (53.98 \pm 9.97). We analyzed the smoking statue without dividing the patients by their gender. There was no significant difference in the proportion of smokers between the three categories (8.2\% in CAE, 9.5\% in CAS, and 6.5\% in normal groups). The table shows that the mean BMI was significantly higher in the CAE group (27.04 \pm 5.57$)$ than in the CAS group $(25.41 \pm$ 5.0). Besides, the prevalence of hyperlipidemia, hypertension, and family history of CAD did not differ between the groups. However, the CAS group was more likely to have diabetes than the CAE group.

\section{Discussion}

Coronary artery ectasia (CAE) is often seen in patients who have undergone coronary angiography due to coronary artery disease. As mentioned in the literature review, the prevalence of ectasia in different populations varies between $3 \%$ and $8 \%$ (6). The current study found that the prevalence of ectasia was $3.04 \%$. It is encouraging to compare this result with those obtained in Turkey (4.3\%), Spain (3.39\%), Greece (2.7\%), the UK (1.4\%), and Singapore (1.2\%). A study by Giannoglou reported it as 1.7\% (7) and Nyamu as 1.9\% (8). A few studies have investigated the prevalence of CAE in Iran. Fariba et al. and Amirzadegan et al. reported the prevalence of CAE as 5.9\% (1) in Hamadan and 2.3\% (5) in Tehran, respectively. The discrepancy in the reported ectasia prevalence in various studies could be attributed to diversities in the general health status of different communities, lifestyles, and various prevalence of cardiovascular diseases.

In reviewing the literature, being a male is a risk factor for ectasia (9). The comparison between the normal group and CAE group in our study indicated that the proportion of males was higher in the ectasia group (48.2\%) than in the normal group (32.3\%); however, the proportion of males was higher in the CAS group (62.1\%) than in the ectasia group (48.2\%). This finding corroborates the research by Fariba (2016) that found that the rate of males with CAE was higher than that of females (69\% vs. 31\%), but there was no significant difference when compared with the normal group (1).

In this study, the mean age of patients with ectasia was significantly higher than that of the normal group (59.44 vs. 53.98 years), which is consistent with the study by Amirzadegan (5). But, this result was not reported by Fariba et al. (1). There were no significant differences in the average age of patients between the ectasia and normal groups. It should be noted that the mean age of the CAS group was higher than that of the ectasia group in the present study (61.19 vs. 59.44) although this difference was not significant. These results do not match those observed in earlier studies by Giannoglou and Bermudez $(7,10)$. A comparison of these results reveals that analytical studies with more robust methodology are required to measure the independent effect of age on the occurrence of ectasia. 


\begin{tabular}{|c|c|c|c|c|c|}
\hline & Normal Group, $(n=744)$ & CAS Group, $(\mathrm{n}=1966)$ & Ectasia Group, $(\mathbf{n}=\mathbf{8 5})$ & P-Value, CAE vs. Normal & P-Value, CAE vs. CAS \\
\hline Age (years) & $53.98 \pm 9.97$ & $61.19 \pm 10.42$ & $59.44 \pm 10.15$ & $<0.001^{\mathrm{a}}$ & 0.136 \\
\hline Sex (male) & $240(32.3)$ & $1220(62.1)$ & $41(48.2)$ & $0.003^{\mathrm{a}}$ & $0.010^{\mathrm{a}}$ \\
\hline $\operatorname{BMI}\left(\mathbf{k g} / \mathbf{m}^{2}\right)$ & $26.15 \pm 5.74$ & $25.41 \pm 5.0$ & $27.04 \pm 5.57$ & 0.086 & $0.004^{\mathrm{a}}$ \\
\hline HDL & $42.30 \pm 13.12$ & $40.18 \pm 13.98$ & $39.69 \pm 12.43$ & $0.028^{\mathrm{a}}$ & 0.598 \\
\hline LDL & $101.64 \pm 34.77$ & $104.27 \pm 36.47$ & $108.56 \pm 38.65$ & 0.562 & 0.412 \\
\hline Chol. & $178.16 \pm 46.90$ & $183.36 \pm 51.10$ & $184.28 \pm 49.68$ & 0.303 & 0.761 \\
\hline TG & $148.63 \pm 97.24$ & $150.77 \pm 90.24$ & $159.09 \pm 87.86$ & 0.175 & 0.263 \\
\hline Hypertension & $224(30.1)$ & $719(36.6)$ & $34(40.0)$ & 0.620 & 0.521 \\
\hline DM & $87(12.9)$ & $489(27.0)$ & $14(18.4)$ & 0.334 & $0.013^{\mathrm{a}}$ \\
\hline Cigarette smoking & $48(6.5)$ & $186(9.5)$ & $7(8.2)$ & 0.531 & 0.705 \\
\hline Family history of CAD & $73(9.8)$ & $161(8.2)$ & $3(3.5)$ & 0.071 & 0.121 \\
\hline
\end{tabular}

${ }^{a}$ P-value was significant.

The results of this study did not show any significant difference in the mean BMI, as well as the prevalence of overweight and obesity between the ectasia and normal coronary groups. But, compared to the CAS group, patients with ectasia were significantly higher in these parameters (average BMI: 27.04 vs. $25.41 \mathrm{~kg} / \mathrm{m}^{2}$ ). The findings of the study concerning BMI are similar to the report by Amirzadegan (average BMI: 29.28 in ectasia and 27.51 in CAD groups) (5). There seems to be an association between BMI and ectasia prevalence, which needs further investigation.

In this study, the prevalence of high BP was $35 \%$ in the total sample, with no statistically significant difference between the three groups (40\% in ectasia, 30.1\% in normal coronary, and $36.6 \%$ in CAS groups). These results agree with the findings of other studies $(11,12)$, but are not consistent with the study by Fariba, in which the prevalence of high blood pressure was reported as 57.7\% in the ectasia group that was significantly higher than in the normal group (1).

The most interesting finding in this study was that the prevalence of dyslipidemia was $80.9 \%$, and there was no significant difference between ectasia with normal coronary and ectasia group with CAS. The prevalence of dyslipidemia in ectasia has been different in various studies. In prior studies, no significant variations have been reported in the prevalence of dyslipidemia between normal, ectasia, and CAS groups $(5,11)$. While the Fariba study reported the prevalence of dyslipidemia as $60 \%$, and a significant difference was observed in dyslipidemia (including hypercholesterolemia and hypertriglyceridemia) between the ectasia and normal groups. Also, the average values of labora- tory parameters, including triglyceride, cholesterol, HDL, and LDL, were investigated separately in the groups. It is somewhat surprising that only HDL levels were significantly lower in the ectasia group than in the normal coronary group $(\mathrm{P}=0.028)$, which is consistent with the study by Sudhir (13).

This study demonstrated that the prevalence of diabetes mellitus was $23 \%$, with no significant difference between the normal and ectasia groups (12.9\% vs. $18.4 \%)$. Also, the prevalence of diabetes was significantly higher in the CAS group (27\%) than in the ectasia group (18.4\%). This study produced results that corroborate the findings of many previous studies, including the studies by Ginnoglou, Obaid, and Amirzadegan that showed a negative correlation between diabetes and $\operatorname{CAE}(5,7,11)$. In another study by Huang, diabetes was presented as a protective factor against ectasia (14).

The overall frequency of smoking was $8.6 \%$ in this study. According to our results, there was no significant difference in smoking between the three groups. This finding contrasts the results by Fariba and Amirzadegan that showed the prevalence of smoking was significantly higher in the ectasia group than in the normal coronary group. It seems that this result was due to the higher proportion of males in the ectasia group who tend more to smoking. Ramezani findings also accord with our observations that showed no differences in smoking between ectasia (33\%) and control groups (22.6\%) (12). It seems that in our study population, most people were likely to deny smoking, and for this reason, there was no difference between the different groups under investigation. 
According to the results, there was no significant difference in the family history of cardiovascular diseases between the three groups, which is similar to the result by Amirzadegan (5).

\subsection{Conclusion}

The evidence from this study suggests no relationship between ectasia and most cardiovascular risk factors, except for the negative relationship between ectasia and diabetes and a positive relationship between low HDL and ectasia. Further investigations in other provinces of the country are strongly recommended to provide more knowledge of clinical and epidemiological factors affecting CAE in Iran.

\subsection{Limitation}

Given that this retrospective study was done by reviewing the data recorded previously, the information of some patients was incomplete.

\section{Footnotes}

Authors' Contribution: Study concept and design: T.K and E.R; Analysis and interpretation of data: S.KH; Drafting of the manuscript: SA.M; Critical revision of the manuscript for important intellectual content: N.A; Statistical analysis: S.KH.

Conflict of Interests: The authors declare no conflict of interest.

Ethical Approval: Ethical approval was obtained (Ir.bums.REC.1396.170).

Funding/Support: This study was funded by the Birjand University of Medical Sciences.

Informed Consent: Informed consent was obtained.

\section{References}

1. Fariba F, Moradi M, Arabi A, Ghaderi B. Prevalence of coronary artery ectasia with atherosclerosis and associated risk factors in the west of Iran: a cross-sectional study. J Res Health Sci. 2016;16(1):22-5.

2. Elguindy MS, Elguindy AM. Aneurysmal coronary artery disease: An overview. Glob Cardiol Sci Pract. 2018;2017(3). doi: 10.21542/gcsp.2017.26. [PubMed: 29564347].

3. Manginas A, Cokkinos DV. Coronary artery ectasias: imaging, functional assessment and clinical implications. Eur Heart J. 2006;27(9):1026-31. doi:10.1093/eurheartj/ehi725. [PubMed: 16415301]

4. Antoniadis AP, Chatzizisis YS, Giannoglou GD. Pathogenetic mechanisms of coronary ectasia. Int J Cardiol. 2008;130(3):335-43. doi: 10.1016/j.ijcard.2008.05.071. [PubMed:18694609].

5. Amirzadegan AR, Davoodi G, Soleimani A, Tokaldany ML, Kazazi EH, Shabpiray $\mathrm{H}$, et al. Association between traditional risk factors and coronary artery ectasia: a study on 10057 angiographic procedures among iranian Population. J Tehran Univ Heart Cent. 2014;9(1):27.

6. Mann DL, Zipes DP, Libby P. Braunwald's heart disease: a textbook of car diovascular medicine. Amsterdam, Netherlands: Elsevier; 2015.

7. Giannoglou GD, Antoniadis AP, Chatzizisis YS, Damvopoulou E, Parcharidis GE, Louridas GE. Prevalence of ectasia in human coronary arteries in patients in northern Greece referred for coronary angiography. Am J Card. 2006;98(3):314-8. doi: 10.1016/j.amjcard.2006.02.034.

8. Kruger D, Stierle U, Herrmann G, Simon R, Sheikhzadeh A. Exerciseinduced myocardial ischemia in isolated coronary artery ectasias and aneurysms ("dilated coronaropathy"). I Am Coll Cardiol. 1999;34(5):1461-70. doi:10.1016/s0735-1097(99)00375-7.

9. Almansori MA, Elsayed HA. Coronary artery ectasia - A sample from Saudi Arabia. J Saudi Heart Assoc. 2015;27(3):160-3. doi: 10.1016/j.jsha.2015.03.005. [PubMed: 26136630].

10. Pinar Bermudez E, Lopez Palop R, Lozano Martinez-Luengas I, Cortes Sanchez R, Carrillo Saez P, Rodriguez Carreras R, et al. [Coronary ectasia: prevalence, and clinical and angiographic characteristics]. Rev Esp Cardiol. 2003;56(5):473-9. doi:10.1016/s0300-8932(03)76902-4.

11. Abid A, Jalala S, Gad M, Gehani A. Clinical and angiographic features of patients with coronary artery ectasia compared with stenotic coronary artery disease. J Cardiol Curr Res. 2017;9(4). doi: 10.15406/jccr.2017.09.00328.

12. Ramezani J, Moghiman T, Azad F, Ghasemi G, Ahmadi M, Shabestari M. Coronary risk factors in patients with coronary artery ectasia : A case-control study from Iran. Biosci Biotechnol Res Asia. 2016;13(1):4016. doi: $10.13005 / \mathrm{bbra} / 2047$.

13. Sudhir K, Ports TA, Amidon TM, Goldberger IJ, Bhushan V, Kane JP, et al. Coronary heart disease/platelet activation/myocardial infarction: Increased prevalence of coronary ectasia in heterozygous familial hypercholesterolemia. Circulation. 1995;91(5):1375-80. doi: 10.1161/01.Cir.91.5.1375. [PubMed: 7867176].

14. Huang Q, Liu J, Chen M, Li J. Relation of diabetes to coronary artery ectasia: A meta-analysis study. Anatol J Cardiol. 2014;14(4):322-7. doi: 10.5152/akd.2014.5327. [PubMed: 24818774]. 